\title{
Adhesion Mechanism of Water Droplets on Hierarchically Rough Superhydrophobic Rose Petal Surface
}

\author{
Hannu Teisala, Mikko Tuominen, and Jurkka Kuusipalo \\ Paper Converting and Packaging Technology, Department of Energy and Process Engineering, Tampere University of Technology, \\ P.O. Box 541, 33101 Tampere, Finland
}

Correspondence should be addressed to Hannu Teisala, hannu.teisala@tut.fi

Received 10 March 2011; Revised 19 April 2011; Accepted 27 April 2011

Academic Editor: Hongchen Chen Gu

Copyright ( $) 2011$ Hannu Teisala et al. This is an open access article distributed under the Creative Commons Attribution License, which permits unrestricted use, distribution, and reproduction in any medium, provided the original work is properly cited.

Extremely hydrophobic surfaces, on which water droplets sit in a spherical shape leaving air entrapped into the roughness of the solid, are often called superhydrophobic. Hierarchically rough superhydrophobic surfaces that possess submicron scale fine structures combined with micron scale structures are generally more hydrophobic, and water droplet adhesion to those surfaces is lower in comparison with surfaces possessing purely micrometric structures. In other words, usually a fine structure on a superhydrophobic surface reduces liquid-solid contact area and water droplet adhesion. Here we show that this does not apply to a highadhesive superhydrophobic rose petal surface. Contrary to the present knowledge, the function of the fine structure on the petal surface is to build up the high adhesion to water droplets. Understanding of the specific adhesion mechanism on the rose petal gives insight into an interesting natural phenomenon of simultaneous superhydrophobicity and high water droplet adhesion, but, in addition, it contributes to more precise comprehension of wetting and adhesion mechanisms of superhydrophobic surfaces overall.

\section{Introduction}

Superhydrophobic surfaces have attracted enormous scientific interest during the last decade [1-16]. Those extremely water-repellent surfaces are not only scientifically interesting, but also because they can be potentially utilised in variety of applications [1-13], including self-cleaning surfaces, aircraft technology, droplet transportation, windscreens, and microand macrofluidic devices. However, despite the intensive study, especially the adhesion mechanisms of superhydrophobic surfaces are not yet completely understood. Although it is well known that an increase in the liquid-solid contact area between a water droplet and a superhydrophobic surface contributes to increase in the droplet adhesion to the surface $[5,9-11,15,17]$, the mechanisms of simultaneous superhydrophobicity and extremely high water droplet adhesion are not well comprehended.

Superhydrophobic surfaces found in nature have guided us to understand the mechanisms and surface structures behind the extreme water repellency. Hydrophobic plants are known to have waxy and comparatively low-energetic surface. Submicron and micron scale roughness of those surfaces can further increase their hydrophobicity significantly $[4-7,9-11,17]$. The most well-known example of superhydrophobicity is the leaf of the lotus plant [17], on which especially the submicron scale fine structure of the hierarchically rough surface increases the hydrophobicity and reduces liquid-solid contact area and thus water droplet adhesion to the surface.

Another hierarchically rough superhydrophobic surface found in nature is the rose petal, but in contrast to the lotus surface, it can create high adhesion to water droplets. However, so far the adhesion mechanism on the rose petal surface is not completely understood. There is only a hypothesis $[6,18-20]$, according to which the submicron scale fine structure of the surface would entrap air and cause the superhydrophobicity, whereas water penetration into the micrometric roughness of the surface would cause the high adhesion of water droplets. Our study reveals that water does not penetrate into the micrometric roughness of the surface as the present hypothesis suggests. Contrary to the lotus surface, the function of the fine structure on the 


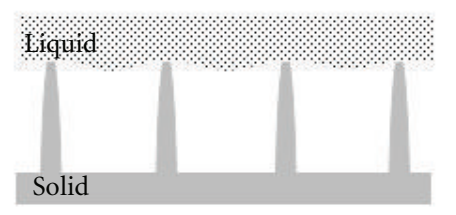

(a)

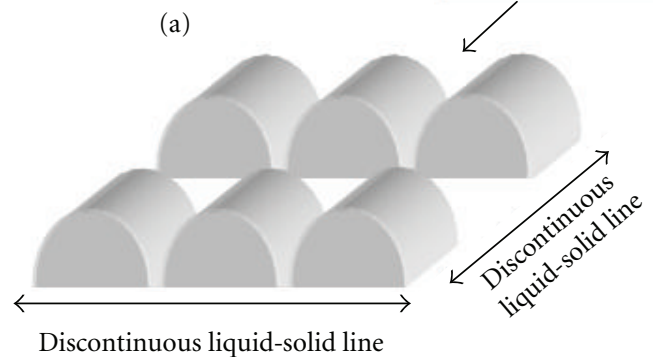

(d)

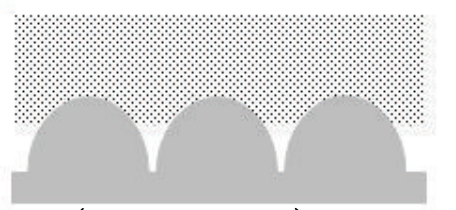

(b)
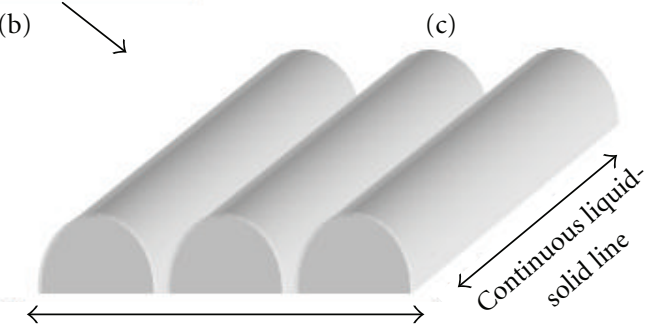

Discontinuous liquid-solid line

(e)

Figure 1: Shape of the fine structures on superhydrophobic surfaces affect the liquid-solid contact area and water droplet adhesion. A spikylike structure reduces the liquid-solid contact area effectively (a). When the structure gets rounder the liquid-solid contact area increases $(\mathrm{a}-\mathrm{c})$, and can become much larger in comparison with the smooth surface. Nubby structure (d) has remarkably smaller liquid-solid contact area compared to grooved structure (e).

high-adhesive rose petal is to build up the high water droplet adhesion.

\section{Materials and Methods}

Fresh roses were purchased from Keinupuiston Kukka, Tampere, Finland. Drying of the roses was performed by pinning the petals, after which they were dried for 7 days in a conditioned room $\left(23^{\circ} \mathrm{C} / 50 \% \mathrm{RH}\right)$ and 3 more days in exsiccator.

Optical microscope (Zeiss Axioskop 40) was used for imaging the surfaces. The petals were immersed in oil and were set between two laboratory glass plates prior to imaging the surfaces from above. For the side imaging, the petals were folded between the laboratory glasses. Prior to the scanning electron microscope imaging (Philips XL-30) the dried petals were sputter coated by thin layers of gold.

Water contact angles were measured in a conditioned room $\left(23^{\circ} \mathrm{C} / 50 \% \mathrm{RH}\right)$ using KSV CAM200 equipment. Three parallel droplets of distilled water were used for each contact angle measurement. In order to determine the tilting angle required for water droplet rolloff, the droplets $(10 \mu \mathrm{L})$ were gently deposited onto the substrate, which was then slowly tilted until the rolloff occurred. Contact angle hysteresis on the surface was studied by imaging the rolling droplets $(10 \mu \mathrm{L})$ on the tilted substrate (at angle of $9^{\circ}$ ). Contact angle, contact diameter, and volume of evaporating droplets were followed by imaging the droplet every $60 \mathrm{~s}$.

\section{Results and Discussion}

Recent studies [6, 18-20] suggest that liquid penetration into the large-scale roughness, that is, between the epidermal cells that appear as micrometric nubs on the rose petal, would cause the high adhesion of water droplets to the surface. However, concrete evidences of that type of wetting have not been shown. The proposed water collapse into the structure has been explained by large gaps, typically around $10-20 \mu \mathrm{m}$, between the nubs of the surface. The size, shape, and chemistry of the surface structures, as well as the droplet size, always affect the critical point where the collapse takes place. Still, studies with superhydrophobic micropillar surfaces $[13,21,22]$ have shown that the air gaps required for water collapse into the structure are usually much larger than the gaps on the rose petal. It is not unusual that the gaps between the pillars must be increased to larger than $100 \mu \mathrm{m}$ until the water collapse occurs.

Less attention has been paid to the geometry of the submicron scale cuticular folding on the high-adhesive rose petals, which forms round and grooved fine structure that is oriented downwards from the nubs of the petal surface $[4,6,18,19]$. Generally, including the rose petal surface, a fine structure on a hierarchically rough surface is considered to decrease the liquid-solid contact area and increase the hydrophobicity of the surface $[5,10,11,17,19,20]$. This is relevant when the fine structure geometry is sharp, for example, tubular or spiky-like (Figure 1(a)), which is the case of the lotus $[4-6,17]$ and lotus-like surfaces. Because of the discontinuous liquid-solid contact, the droplet contact area and adhesion to those surfaces are small. However, when the fine structure gets rounder, the liquid-solid contact area increases gradually and can become even larger in comparison with a completely smooth surface (Figures 1(a), $1(\mathrm{~b})$, and $1(\mathrm{c}))$. When the fine structure is grooved, the liquid-solid contact area can increase further compared to nubby structure (Figures $1(\mathrm{~d})$ and $1(\mathrm{e})$ ) because the contact line is continuous in the direction of the grooves. Therefore, the relatively round and grooved fine structure on the plump micron scale nubs of the rose petal surface can form large enough liquid-solid contact area for the high water droplet adhesion to occur. 


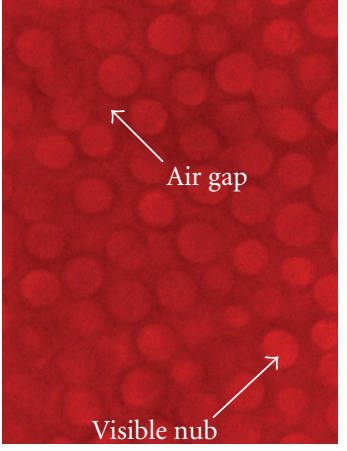

(a)

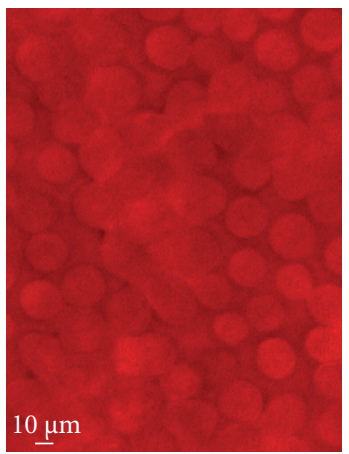

(e)

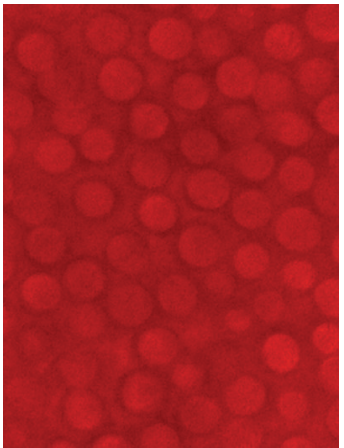

(b)

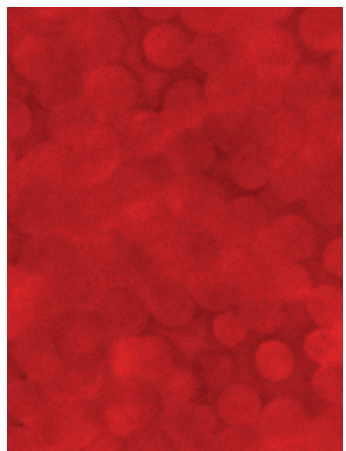

(f)

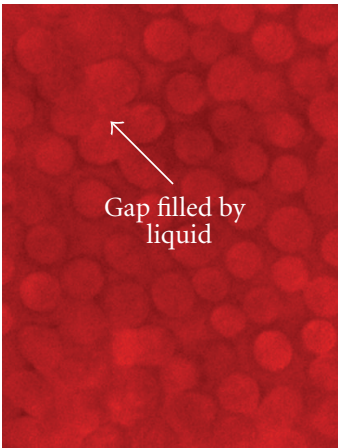

(c)

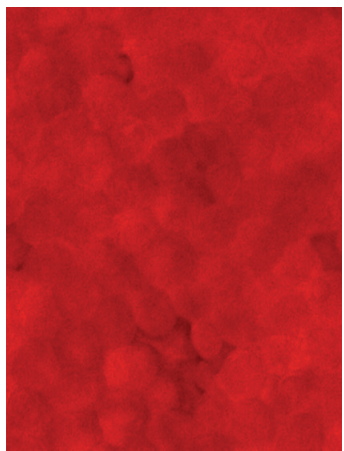

(g)

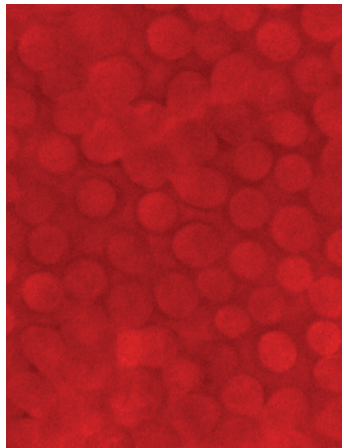

(d)

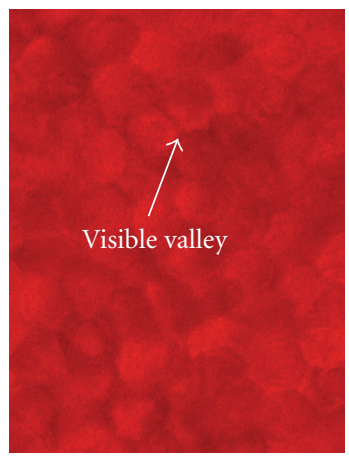

(h)

Figure 2: Liquid film on the fresh rose petal surface. Air pockets remain under the water film in the gaps between the nubs of the surface (a). Top areas of the nubs are visible, but light reflection on the liquid-air interface hinders the view between the nubs. With the decreasing surface tension of the fluid, the air pockets get gradually filled by the liquid and also the valleys between the nubs become visible (b-h).

We set up an experiment to show that on a highadhesive red rose petal (Prestige) water does not fill the space between the micrometric nubs of the surface, but a large amount of air gets entrapped into that space. A water droplet was deposited onto the fresh rose petal, which was then inserted between two laboratory glass plates and imaged by optical microscope. Despite the petal was under a forced wetting between the glass plates, water did not fill the space between the nubs of the surface, but only top areas of the nubs were wetted and are visible in Figure 2(a). In order to obtain a full wetting of the petal surface, the surface tension of water was decreased by introducing a small amount, approximately $20 \mu \mathrm{L}$, of ethanol-water solution $(50 / 50 \mathrm{wt} \%)$ between the glass plates. As the solution was injected between the plates, where it slowly mixed with water and thus gradually decreased the surface tension, the air pockets on the surface were replaced by the liquid one after the other (Figures 2(b)-2(h)). Consequently, also the valleys between the nubs became visible because light reflection on the liquid-air interface no longer disturbed the view. On the basis of the observations in Figure 2, an illustration of the wetting mechanism and formation of the liquid-solid-air composite interface on the rose petal surface is presented in Figure 3. Despite most of the entrapped air exists between the micron scale nubs of the surface, we assume that some air also remains in the folds of the fine structure.

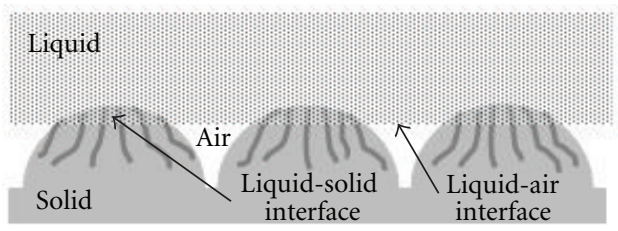

Figure 3: Wetting and adhesion mechanism on the high-adhesive superhydrophobic rose petal surface. Air gets entrapped between the micrometric nubs of the surface. Formation of the liquid-solidair composite interface is essential for superhydrophobicity of the surface. Grooved fine structure on top of the nubs anchors water droplets tightly to the surface.

The grooved fine structure on the plump nubs of the fresh rose petal surface can form large liquid-solid contact area and high water droplet adhesion, as was discussed earlier. However, drying of the petal significantly changes its topography as the micrometric nubs on the surface shrink with the decreasing water content of the cells (Figures 4(a)$4(\mathrm{f}))$. The shrunken nubs appear rather in a pillar-like shape with a relatively sharp head, and therefore, top surface area on those nubs is much smaller in comparison with the plump nubs of the fresh petal. We also observed that on the dried petal the hydrophobicity increased, and water droplet adhesion to the surface decreased in comparison with the 


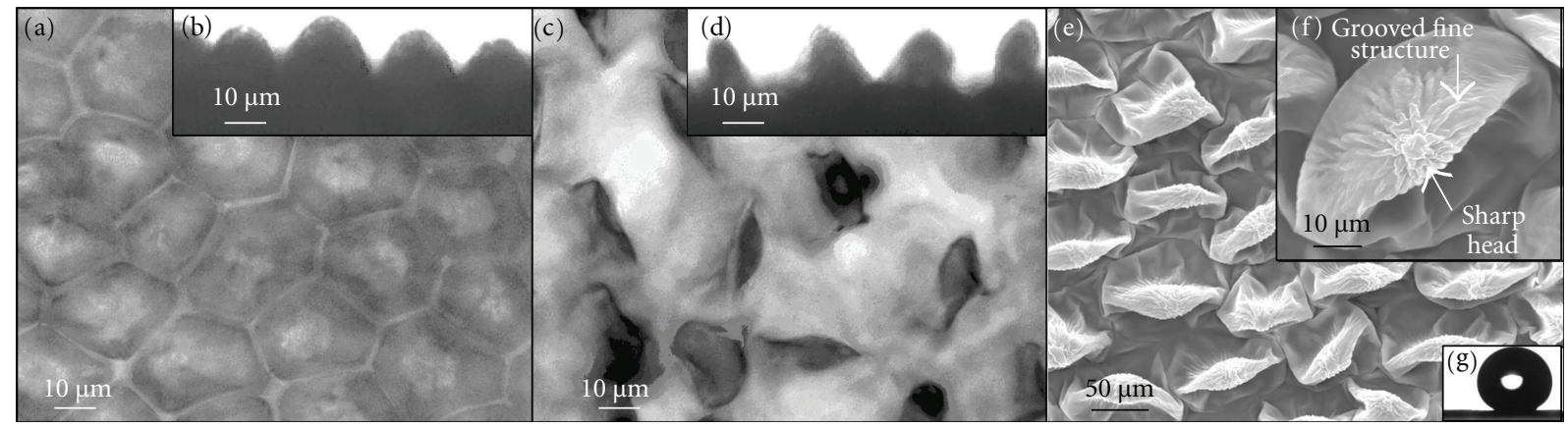

Figure 4: Microscope images of rose petal surfaces. Optical microscope images from above and side of the plump (a, b) and shrunken (c, d) nubs of the fresh and dried petals, respectively. Base diameter of the nubs is typically $20-30 \mu \mathrm{m}$. Round top area of the plump nubs, which is expected to contact with water droplets, is around $10 \mu \mathrm{m}$ in diameter. Top area of the shrunken nubs is significantly smaller in comparison with the plump nubs, and thus the air gaps between the shrunken nubs are larger. Scanning electron microscope images of the dried petal surface $(e, f)$ show that the grooved fine structure exists only on top of the nubs, not at the areas between them. The shrunken nubs possess sharp heads (f), on which a water droplet sits in a spherical shape (g) with small liquid-solid contact area.

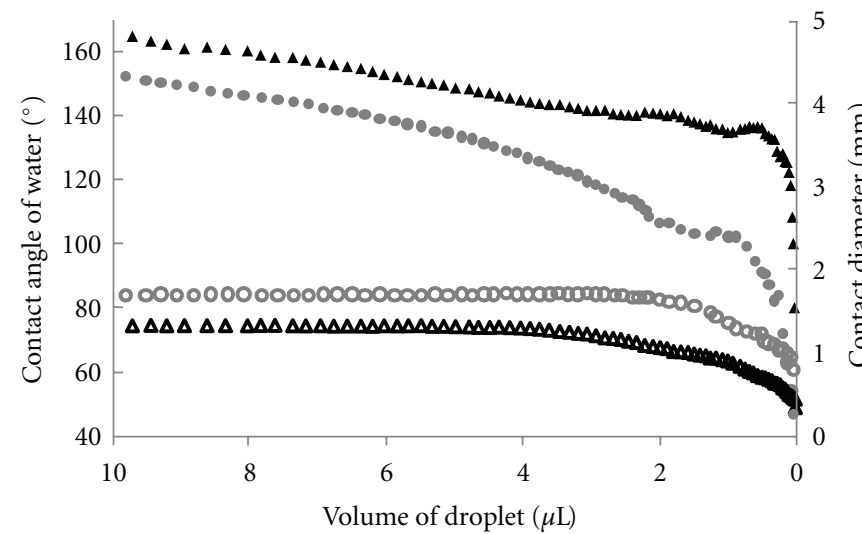

(a)

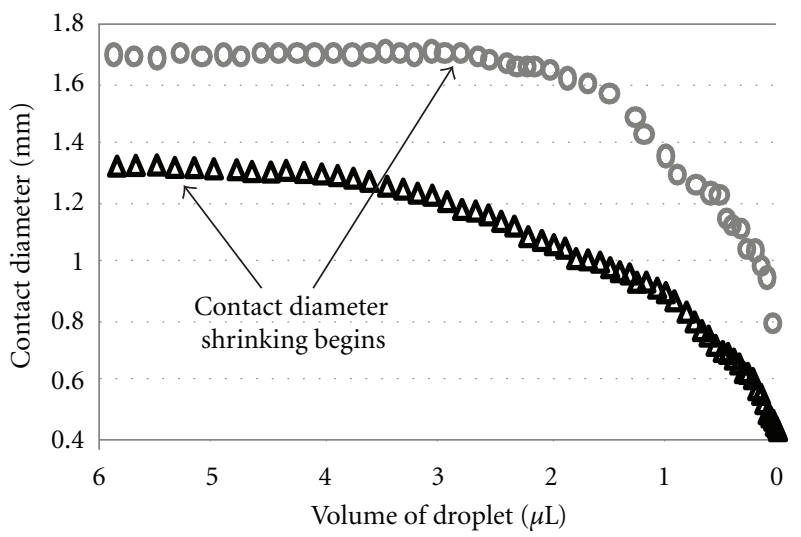

(b)

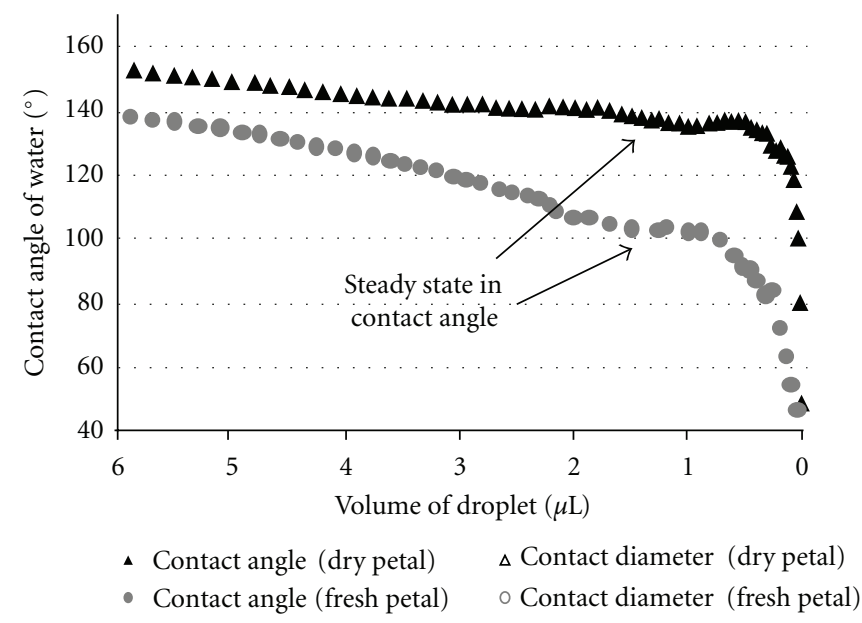

(c)

FIGURE 5: Contact angle and contact diameter of evaporating water droplets on the fresh and dried rose petal surface. The contact angle remains high on the low-adhesive dried petal because the contact diameter shrinks quite freely with the decreasing droplet volume, but on the high-adhesive fresh petal it decreases quite fast because the contact diameter remains pinned (a). The shrinking of the contact diameter begins when the droplet volume on the dried surface is still over $5 \mu \mathrm{L}$, but on the fresh surface it does not begin until the volume is less than $3 \mu \mathrm{L}$ (b). Because of the decreasing contact diameter, the receding contact angle remains approximately constant between the droplet volumes of 2 and $1 \mu \mathrm{L}$, and is $138 \pm 1^{\circ}$ and $104 \pm 1^{\circ}$ on the dried and fresh petal, respectively (c). 
fresh petal. Those are natural consequences related to the drying-induced changes in the surface topography, that is, the liquid-solid contact area on the nubs has decreased, and the air gaps between the nubs have grown larger. In the case that water would fill the space between the nubs of the surface, the above-mentioned drying-induced changes in the hydrophobicity and droplet adhesion would rather be converse. In other words, although the air gaps between the nubs did grow from approximately $10-20 \mu \mathrm{m}$ (Figures 4(a) and 4(b)) to even larger than $20 \mu \mathrm{m}$ (Figure 4(c), 4(d), and $4(\mathrm{e})$ ) due to drying, water did not collapse into the gaps.

Water droplet $(10 \mu \mathrm{L})$ contact angles of $153 \pm 1^{\circ}$ and $165 \pm 1^{\circ}$ were measured on the fresh and dried petal surface, respectively. The droplets were able to remain on the fresh petal even when the surface was tilted upside down. In contrast, from the dried petal the droplets rolled off when the tilting angle exceeded $10^{\circ}$. Contact angle hysteresis, that is, a difference between advancing and receding angles, is only $10 \pm 2^{\circ}$ for a moving droplet on the dried petal. The easy rolloff effect and low contact angle hysteresis on the dried petal surface indicate that the water droplets sit on top of the shrunken nubs with a minimal liquid-solid contact area $[5,10,11,17]$.

Water droplet pinning to the petal surface can easily be illustrated by following a liquid-solid contact diameter [23] of an evaporating droplet (Figure 5(a)). Pinning of the droplet contact diameter is obvious on the high-adhesive fresh petal, because shrinking of the contact diameter does not begin until the droplet has diminished significantly and has the volume of less than $3 \mu \mathrm{L}$ (Figure $5(\mathrm{~b})$ ). Because of the pinned contact diameter, the contact angle of the droplet decreases comparatively fast with the decreasing droplet volume (Figure 5(c)). In contrast, because of the smaller liquid-solid contact area and thus lower adhesion of the droplet to the dried petal, the contact diameter shrinks quite freely with the decreasing droplet volume (Figure 5(b)), and therefore, the droplet is able to maintain its spherical shape and high contact angle around $138^{\circ}$ (Figure 5(c)) until internal Laplace pressure of the droplet grows too high $[13,16,24]$. There is also a comparatively steady state in the contact angle on the fresh petal, as the receding angle remains approximately constant around $104^{\circ}$ between the droplet volumes of 2 and $1 \mu \mathrm{L}$, after which the increasing Laplace pressure causes the rapid decrease in the contact angle. Laplace pressure is inversely proportional to the droplet radius, and therefore it more than doubles when the droplet size decreases from the initial volume of $10 \mu \mathrm{L}$ to $1 \mu \mathrm{L}$, and almost triples when the volume decreases to $0.5 \mu \mathrm{L}$. At certain point, below the droplet volume of $1 \mu \mathrm{L}$, the Laplace pressure overcomes the capillary pressure that prevents water collapse between the micrometric nubs of the surface, and therefore, with very small droplets there is a possibility that water will fall into the micrometric structure.

The wetting mechanism of the high-adhesive superhydrophobic rose petal surface illustrated in this paper gives insight into the interesting natural phenomenon, but, in addition, it brings new information to our present knowledge of superhydrophobicity, especially for high-adhesive surfaces $[6,23,25-27]$. A detailed understanding of super- hydrophobicity and adhesion mechanisms is significant, not only scientifically, but also in designing superhydrophobic surfaces for practical applications. As we have shown here, a fine structure on a hierarchically rough superhydrophobic surface does not necessarily reduce the liquid-solid contact area. That is, not only the size of the surface structures, but also their shape, affects the liquid-solid contact area and droplet adhesion.

\section{Conclusions}

We have shown that on hierarchically rough high-adhesive superhydrophobic rose petal surface water does not fill the gaps between the micrometric nubs of the surface as the earlier studies have hypothesised. Instead, water droplets adhere to the submicron scale fine structure on top of those micrometric nubs. The function of the fine structure on the rose petal is therefore contrary to the lotus or lotus-like surfaces, where the fine structure reduces the liquid-solid contact area and water droplet adhesion. Our study gives insight into an interesting natural phenomenon of simultaneous superhydrophobicity and high droplet adhesion, but, in addition, it contributes to more precise comprehension of wetting and adhesion mechanisms of superhydrophobic surfaces overall.

\section{Acknowledgments}

Mrs. Mari Honkanen, Department of Materials Science, TUT, is acknowledged for the SEM images. The authors would also like to thank Keinupuiston Kukka (Tampere) for the smooth delivery of the roses.

\section{References}

[1] M. Ma and R. M. Hill, "Superhydrophobic surfaces," Current Opinion in Colloid and Interface Science, vol. 11, no. 4, pp. 193202, 2006.

[2] P. Roach, N. J. Shirtcliffe, and M. I. Newton, "Progess in superhydrophobic surface development," Soft Matter, vol. 4, no. 2, pp. 224-240, 2008.

[3] A. Carré and K. L. Mittal, Eds., Superhydrophobic Surfaces, VSP/Brill, Leiden, The Netherlands, 2009.

[4] K. Koch, B. Bhushan, and W. Barthlott, "Multifunctional surface structures of plants: an inspiration for biomimetics," Progress in Materials Science, vol. 54, no. 2, pp. 137-178, 2009.

[5] K. Koch and W. Barthlott, "Superhydrophobic and superhydrophilic plant surfaces: an inspiration for biomimetic materials," Philosophical Transactions of the Royal Society A, vol. 367, no. 1893, pp. 1487-1509, 2009.

[6] M. Liu, Y. Zheng, J. Zhai, and L. Jiang, "Bioinspired superantiwetting interfaces with special liquid-solid adhesion," Accounts of Chemical Research, vol. 43, no. 3, pp. 368-377, 2010.

[7] K. Liu, X. Yao, and L. Jiang, "Recent developments in bioinspired special wettability," Chemical Society Reviews, vol. 39, no. 8, pp. 3240-3255, 2010.

[8] H. Y. Erbil, A. L. Demirel, Y. Avci, and O. Mert, "Transformation of a simple plastic into a superhydrophobic surface," Science, vol. 299, no. 5611, pp. 1377-1380, 2003. 
[9] A. Marmur, "The lotus effect: superhydrophobicity and metastability," Langmuir, vol. 20, no. 9, pp. 3517-3519, 2004.

[10] B. Bhushan and Y. C. Jung, "Wetting, adhesion and friction of superhydrophobic and hydrophilic leaves and fabricated micro/nanopatterned surfaces," Journal of Physics Condensed Matter, vol. 20, no. 22, Article ID 225010, 2008.

[11] B. Bhushan, Y. C. Jung, and K. Koch, "Self-cleaning efficiency of artificial superhydrophobic surfaces," Langmuir, vol. 25, no. 5, pp. 3240-3248, 2009.

[12] N. A. Patankar, "On the modeling of hydrophobic contact angles on rough surfaces," Langmuir, vol. 19, no. 4, pp. 12491253, 2003.

[13] K. K. Varanasi, T. Deng, M. F. Hsu, and N. Bhate, "Wetting hysteresis, metastability, and droplet impact on superhydrophobic surfaces," in Proceedings of the ASME InterPack Conference (IPACK '09), pp. 623-630, San Francisco, Calif, USA, July 2009.

[14] A. Tuteja, W. Choi, M. Ma et al., "Designing superoleophobic surfaces," Science, vol. 318, no. 5856, pp. 1618-1622, 2007.

[15] C. Dorrer and J. Rühe, "Advancing and receding motion of droplets on ultrahydrophobic post surfaces," Langmuir, vol. 22, no. 18, pp. 7652-7657, 2006.

[16] M. Callies and D. Quéré, "On water repellency," Soft Matter, vol. 1, no. 1, pp. 55-61, 2005.

[17] W. Barthlott and C. Neinhuis, "Purity of the sacred lotus, or escape from contamination in biological surfaces," Planta, vol. 202, no. 1, pp. 1-8, 1997.

[18] L. Feng, Y. Zhang, J. Xi et al., "Petal effect: a superhydrophobic state with high adhesive force," Langmuir, vol. 24, no. 8, pp. 4114-4119, 2008.

[19] B. Bhushan and E. K. Her, "Fabrication of superhydrophobic surfaces with high and low adhesion inspired from rose petal," Langmuir, vol. 26, no. 11, pp. 8207-8217, 2010.

[20] B. Bhushan and M. Nosonovsky, "The rose petal effect and the modes of superhydrophobicity," Philosophical Transactions of the Royal Society A, vol. 368, no. 1929, pp. 4713-4728, 2010.

[21] Y. C. Jung and B. Bhushan, "Wetting transition of water droplets on superhydrophobic patterned surfaces," Scripta Materialia, vol. 57, no. 12, pp. 1057-1060, 2007.

[22] C. I. Park, H. E. Jeong, S. H. Lee, H. S. Cho, and K. Y. Suh, "Wetting transition and optimal design for microstructured surfaces with hydrophobic and hydrophilic materials," Journal of Colloid and Interface Science, vol. 336, no. 1, pp. 298-303, 2009.

[23] S. A. Kulinich and M. Farzaneh, "Effect of contact angle hysteresis on water droplet evaporation from super-hydrophobic surfaces," Applied Surface Science, vol. 255, no. 7, pp. 40564060, 2009.

[24] C. Luo, M. Xiang, X. Liu, and H. Wang, "Transition from Cassie-Baxter to Wenzel States on microline-formed PDMS surfaces induced by evaporation or pressing of water droplets," Microfluidics and Nanofluidics, vol. 10, no. 4, pp. 831-842, 2011.

[25] M. Jin, X. Feng, L. Feng et al., "Superhydrophobic aligned polystyrene nanotube films with high adhesive force," Advanced Materials, vol. 17, no. 16, pp. 1977-1981, 2005.

[26] S. Wang and L. Jiang, "Definition of superhydrophobic states," Advanced Materials, vol. 19, no. 21, pp. 3423-3424, 2007.

[27] H. Teisala, M. Tuominen, M. Aromaa et al., "Development of superhydrophobic coating on paperboard surface using the Liquid Flame Spray," Surface and Coatings Technology, vol. 205, no. 2, pp. 436-445, 2010. 

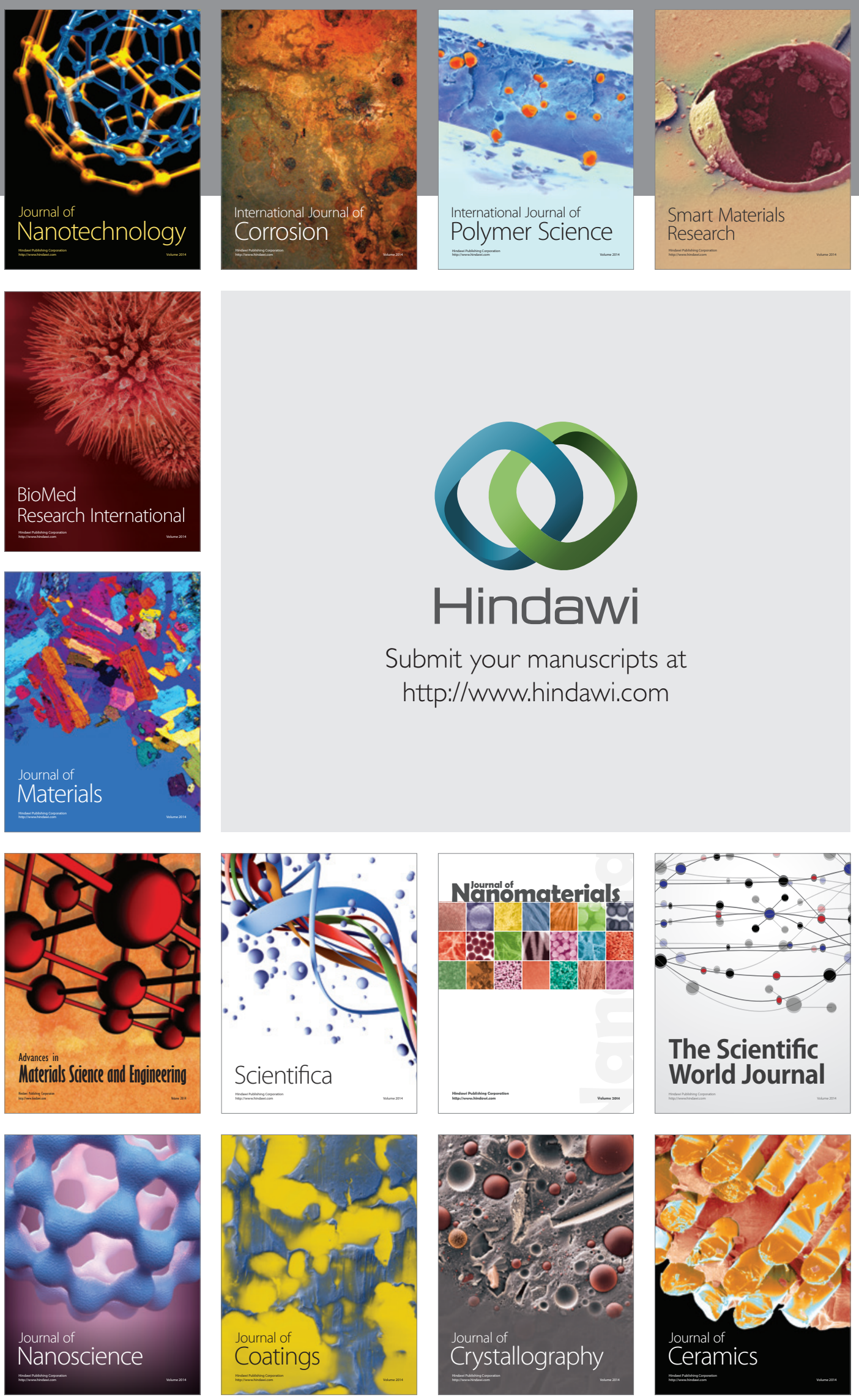

The Scientific World Journal

Submit your manuscripts at

http://www.hindawi.com

\section{World Journal}

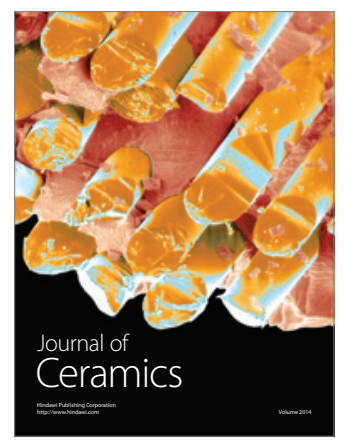

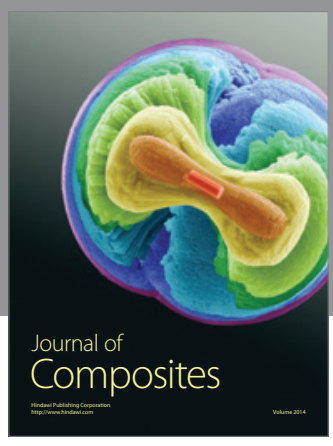
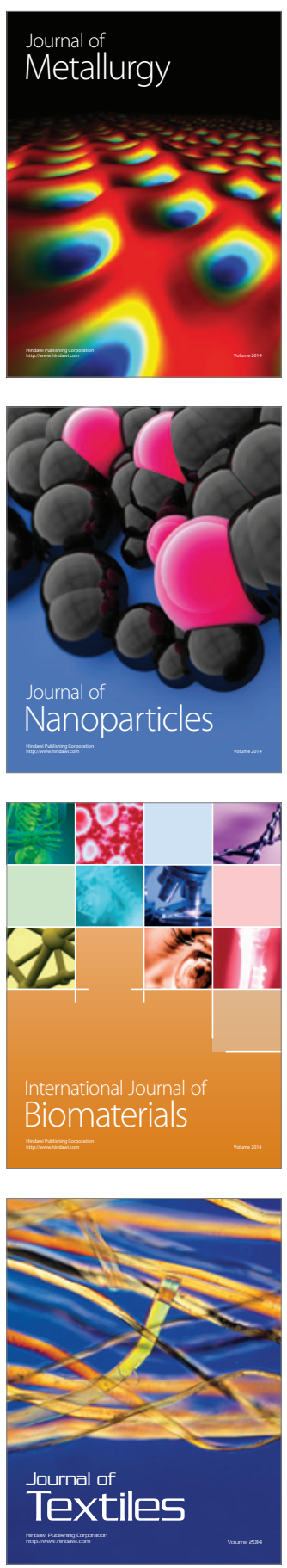